\title{
Abschätzung der Einträge von polyzyklischen aromatischen Kohlenwasserstoffen in deutsche Gewässer
}

\author{
Aktuelle Datengrundlage
}

\author{
Thomas Götz • Thomas Hillenbrand · Frank Marscheider-Weidemann · Stephan Fuchs • Ulrike Scherer
}

Eingegangen: 4. Februar 2009/Akzeptiert: 18. August 2009/Online veröffentlicht: 11. September 2009

(C) Springer-Verlag 2009

Zusammenfassung Hintergrund und Ziel Polyzyklische aromatische Kohlenwasserstoffe (PAK) entstehen in großen Mengen bei unvollständiger Verbrennung aus praktisch allen organischen Stoffen, z. B. bei Waldbränden, durch Hausfeuerungen, in Verbrennungsmotoren und beim Grillen oder Rauchen. PAK werden überwiegend über den Luftpfad verbreitet, nachdem in der Vergangenheit ihre Nutzung in Produkten stark eingeschränkt oder vollständig verboten wurde. Im Zusammenhang mit der Wasserrahmenrichtlinie werden die Einzelstoffe Anthracen, Fluoranthen und Naphthalin sowie die PAK als Stoffgruppe in der Liste der prioritären Stoffe aufgeführt. Hintergrund der Arbeit ist eine erste Zusammenstellung der PAK-Einträge in Gewässer und in diesem Zusammenhang die Erhebung aller Daten und Informationen zu der Gesamtberechnung in Deutschland.

Material und Methoden Im Rahmen von verschiedenen, durch das Umweltbundesamt geförderten Forschungsvorhaben wurden durch das Fraunhofer Institut für System- und Innovationsforschung aktuelle Daten zu PAK-Einträgen in Gewässer erhoben. Auf Basis der erhobenen Daten wurde

Verantwortlicher Herausgeber: Werner Brack

T. Götz · T. Hillenbrand · F. Marscheider-Weidemann $(\bowtie)$ Fraunhofer-Institut für System- und Innovationsforschung, Breslauer Straße 48, 76139 Karlsruhe, Deutschland

E-Mail: MW@isi.fraunhofer.de

S. Fuchs

Institut für Wasser und Gewässerentwicklung, Bereich

Siedlungswasserwirtschaft und Wassergütewirtschaft,

Universität Karlsruhe (TH),

Adenauerring 20, 76128 Karlsruhe, Deutschland

U. Scherer

Technische Universität München, Institut für Wasser und Umwelt, Arcisstr. 21, 80333 München, Deutschland unter Einsatz des Modells MONERIS eine erste Abschätzung der Relevanz der verschiedenen Quellen durchgeführt.

Ergebnisse PAK gelangen durch die atmosphärische Deposition nicht nur direkt über Wasseroberflächen, sondern auch indirekt durch den Oberflächenabfluss über andere Pfade (z.B. über den Niederschlagsabfluss von urbanen Flächen, Kläranlagen, Erosion) in erheblichem Umfang in Gewässer.

Diskussion Obwohl bei einigen der betrachteten Pfade noch ergänzender Forschungsbedarf besteht, ergibt sich bei der Gesamtbetrachtung eine deutliche Relevanzverteilung.

Schlussfolgerungen Die weitere Entwicklung der PAKEinträge in Gewässer wird wesentlich davon abhängen, inwieweit im Bereich atmosphärischer PAK-Emissionen Maßnahmen zur Emissionsminderung umgesetzt werden. Zusätzlicher Forschungsbedarf besteht vor allem hinsichtlich der Emissionen durch PAK-Belastungen in Produkten, durch Binnenschifffahrt und Sportboote sowie durch den Pfad „Erosion“.

Empfehlungen und Perspektiven Bei der als ,,prioritär gefährlich“ eingestuften PAK-Stoffgruppe sind entsprechend WRRL langfristig alle Einleitungen, Emissionen und Verluste zu beenden oder schrittweise einzustellen. Bis 2015 sind für alle prioritären Stoffe Qualitätsnormen (UQN) einzuhalten. Derzeit werden für viele PAK einschließlich Benzo(a)pyren die Zielvorgaben für Gewässer nur teilweise eingehalten. Emissionsminderungsmaßnahmen für PAK sind in unterschiedlichen Bereichen notwendig, vor allem jedoch im Bereich der diffusen atmosphärischen Einträge.

Schlüsselwörter Atmosphärische Deposition ·

Hausbrand · Holzfeuerungsanlagen · MONERIS · PAK ·

Polyzyklische aromatische Kohlenwasserstoffe ·

Prioritäre Stoffe · Stoffeinträge in Gewässer ·

Wasserrahmenrichtlinie $\cdot$ WRRL 


\section{Deposition of polycyclic aromatic hydrocarbons in surface waters - state of knowledge}

Abstract Background, aim, and scope Polycyclic aromatic hydrocarbons (PAH) result from incomplete combustion of almost every organic materials, e.g. due to forest fires, residential heating, combustion engines, grilling, or smoking. PAH are predominantly distributed over the air-path. Their usage in products (e. g. moth-balls) has been limited strictly or completely forbidden in the past. In the context of the EU Water Framework Directive (WFD), the single substances anthracene, fluoranthene and naphthalene, as well as PAHs as group of substances, are listed as "priority" substances. Background of this work is a first compilation of sources of PAH emissions, and in this context, the collection of all relevant data and information to calculate the total emissions into surface waters in Germany.

Materials and methods Within the scope of diverse research projects, funded by the German Environmental Agency, the available data material concerning PAH input in surface waters has been analyzed by the Fraunhofer Institute for Systems and Innovation Research. On the basis of the collected data, a first evaluation of relevance of the different sources has been conducted using the model MONERIS.

Results As a result of the atmospheric deposition, PAHs enter, to a large extent, surface waters directly or indirectly through surface runoff (e.g. urban areas, wastewater treatment plants, erosion).

Discussion Although there is still additional research need for some of the covered PAH sources, a noticeable distribution pattern of relevance emerges.

Conclusions The scenarios, based on the currently known sources, show that the implementation or the neglect of emission reduction measures in the field of diffuse air-borne PAHs are crucial for the further development of the deposition of PAHs in surface waters. More far-reaching studies are necessary, e.g. concerning emissions from inland navigation/motor boats, the inhomogeneous source "products", or the path "erosion".

Recommendations and perspectives According to the EU-WFD, all depositions, emissions and losses of "priority hazardous" classified substances, such as the group of PAHs, have to be stopped or gradually finished in the long term. Until the year 2015, environmental quality standards (EQS) have to be met for all "priority substances". Presently, the EQS often are not completely fulfilled for many substances of the PAH group, including benzo(a)pyrene. Reduction measures are necessary within diverse areas, especially concerning diffuse atmospheric emissions (e.g. "residential heating").

Keywords Atmospheric deposition · MONERIS · PAHs · Polycyclic aromatic hydrocarbons · Priority substances ·
Residential heating - Substance input into surface waters · Water Framework Directive · WFD · Wood firing plant Wood pellet heating appliance

\section{Problemstellung}

Polyzyklische aromatische Kohlenwasserstoffe (PAKs) sind aus mehreren „kondensierten“ Benzolringen aufgebaute Verbindungen, die teilweise als beim Menschen krebserregend eingestuft sind. Insgesamt sind mehr als 100 Verbindungen bekannt, wie etwa Naphthalin mit zwei Benzolringen, Anthracen und Fluoranthen mit drei Ringen und das häufig als Leitsubstanz verwendete, besonders toxische Benzo(a)pyren mit fünf Ringen. Die Bestimmung der PAKs ist aufgrund der Vielzahl der Einzelverbindungen schwierig, weshalb häufig nur die wichtigsten Vertreter erfasst und als „PAK Summe“ dargestellt werden. Beispiele hierfür sind die Borneff EPAK6 (Benzo(a)pyren, Benzo(g,h,i)perylen, Benzo(k)fluoranthen, Fluoranthen, Indeno(1,2,3-cd) pyren, Benzo(b)fluoranthen) oder nach einem Vorschlag der amerikanischen Umweltbehörde EPA die $\Sigma$ PAK16 mit den zusätzlichen Stoffen Naphthalin, Acenaphthen, Acenaphthylen, Fluoren, Anthracen, Phenanthren, Pyren, Benzo(a)anthracen, Chrysen und Dibenzo(a,h)anthracen. Im Folgenden wird die Datengrundlage für eine erste Gesamtzusammenstellung der wichtigsten punktuellen und diffusen Eintragspfade für PAKs in deutsche Gewässer näher beschrieben. Für eine direkte Vergleichbarkeit wird der EPA-PAK16 Summenparameter betrachtet. Liegen Daten für eine geringere Anzahl an PAKs vor, erfolgt eine Extrapolation. Die hierfür verwendeten Werte werden in Abhängigkeit von der jeweiligen Datenlage bei den einzelnen Eintragspfaden aufgeführt, wobei mit den Extrapolationen verbundene Unsicherheiten bei der Ergebnisinterpretation $\mathrm{zu}$ berücksichtigen sind. Entsprechend BMU (2006) sind bei PAK-Verbindungen im Zeitraum von 2002 bis 2004 an ca. 10 bis $25 \%$ der beprobten Messstellen an deutschen Gewässern Überschreitungen von Umweltqualitätsnormen für den ökologischen und chemischen Zustand nach EU-WRRL festzustellen. Nach einer Länderabfrage bezüglich der Gewässerbelastungssituation für PAKs in Deutschland wurden mehrere PAK-Vertreter sowie PAK als Summenparameter in der Gesamtbewertung als ,,relevant“ eingestuft (Lehmann und Vietoris 2006).

\section{Rechtliche Rahmenbedingungen}

In der Liste der prioritären Stoffe der Wasserrahmenrichtlinie werden sowohl die Einzelstoffe Anthracen, Fluoranthen und Naphthalin als auch die PAK als Stoffgruppe aufgeführt. Aktuell werden in Deutschland nur Anthracen, Naph- 
thalin und, in sehr kleiner Menge, auch Fluoranthen zur Herstellung von Farbstoffen und als Zwischenprodukte eingesetzt. Die Verwendung von Kreosoten im Holzschutz ist stark beschränkt worden und nur noch für gewerbliche und industrielle Anwendungen gestattet. Die wichtigsten Regelungen zur Emissionsbegrenzung sind im Wasserbereich die Abwasserverordnung (Anhang 46, Steinkohleverkokung) sowie im Bereich Luftreinhaltung die Richtlinie 2004/107/ $\mathrm{EG}$, in der neben $\mathrm{Cd}, \mathrm{Hg}$ und $\mathrm{Ni}$ auch PAKs geregelt werden. Die Beschränkungsrichtlinien der EU für Teeröle (2001/90/EG) und von PAKs in Weichmacherölen und Reifen $(2005 / 69 / E G)$ wurden über die ChemikalienverbotsV in deutsches Recht überführt (Hillenbrand et al. 2006).

\section{Datengrundlage zu PAK-Gewässereinträgen}

\subsection{PAK-Emissionen aus Punktquellen}

\subsubsection{Kommunale Kläranlagen}

Die Quantifizierung von Emissionen aus kommunalen Kläranlagen erfolgt in der Regel durch Multiplikation der Stoffkonzentrationen im Kläranlagenablauf mit der behandelten Abwassermenge (Fuchs et al. 2002). Schon die PAK-Zulaufkonzentrationen liegen jedoch bereits häufig unter der Bestimmungsgrenze. Alternativ können Ablaufkonzentrationen durch eine Frachtberechnung auf Basis von Klärschlammdaten ermittelt werden. Die Elimination bei der kommunalen Abwasserbehandlung wird mit 90\% angenommen, wobei die aus dem Abwasser abgetrennten PAK-Verbindungen in den Klärschlamm gelangen (Ivashechkin 2005). Anhand von Erhebungen durch Kollotzek et al. (1996) für Klärschlämme kann ein mittleres Verhältnis von $2,3 \times$ PAK6 $($ TVO $)=\Sigma$ EPA-PAK16 ermittelt werden . Für die Berechnung der Stoffkonzentration im Kläranlagenablauf wird ein mittlerer Gehalt von $\Sigma$ EPA-PAK16 in Klärschlämmen in Deutschland von 5,5 mg/kg TS (UBA 2007b) angenommen. Ausgehend von dem Klärschlammanfall in Deutschland 2004 (BMU 2007) können 12,9t EPA-PAK16 pro Jahr im Zulauf der Kläranlagen in Deutschland berechnet werden, von denen etwa 1,3t pro Jahr in die Gewässer gelangen. Unter Verwendung der in Deutschland 2004 behandelten Abwassermenge (Destatis 2004) ergibt sich eine mittlere Ablaufkonzentration von $0,14 \mu \mathrm{g} / \mathrm{L} \Sigma$ EPA-PAK16.

\subsubsection{Industrielle Einleiter}

Für industrielle Einleiter kann das Europäische Emissionsinventar EPER für das Berichtsjahr 2004 ausgewertet werden (EPER 2007). Dabei gelten als Schwellenwerte, bezogen auf Borneff PAK6, für die Erfassung von Luftemissionen $50 \mathrm{~kg}$ pro Jahr sowie für Gewässereinträge $5 \mathrm{~kg}$ pro Jahr (EPER 2007). Entsprechend Kollotzek et al. (1996) wird ein Verhältnis von EEPA-PAK16 = Borneff PAK6 $\times$ 2,3 angenommen. Die berichteten Einträge aus industrieller Direkteinleitung in Gewässer betragen demnach $443 \mathrm{~kg}$ इEPA-PAK16.

\subsection{PAK-Emissionen aus diffusen Quellen}

\subsubsection{Atmosphärische Deposition auf die Gewässeroberfläche}

PAKs entstehen z.B. bei Waldbränden, durch Hausbrand, in Verbrennungsmotoren und beim Grillen oder Rauchen bei unvollständiger Verbrennung aus praktisch allen organischen Stoffen und werden deshalb vorherrschend über den Luftpfad verbreitet (Gocht und Gratwohl 2004). In der Literatur zur umweltchemischen Analytik der PAKs wird seit langem untersucht, inwieweit durch die unterschiedliche Anwesenheit und Konzentration der PAK-Vertreter (ihrer „fingerprints“) z.B. in Sedimenten auf die Emissionsquellen, wie direkte Öleinträge in Gewässer, oder auf bestimmte einzelne verbrannte Energieträger wie Heizöle oder Kohle geschlossen werden kann (Blumer 1976). Diese Methodik hat sich verfeinert, und es liegen für einzelne Flussgebiete aus flächendeckenden PAK-Messungen gute Aussagen für die Eintragsquellen vor, siehe beispielsweise Yunker et al. (2002). Für Deutschland haben sich aus der Literatur aber derzeit noch keine Ansatzpunkte für die Berechnung von Einträgen in Gewässer ergeben. Literaturangaben zur resultierenden PAK-Deposition sind durch starke Schwankungen (Faktor 10 bis 1000) gekennzeichnet (Götz 2008). Wesentliche Ursachen sind dabei variierende Bestimmungsmethoden (Sammlertechnik und Anzahl untersuchter PAKKongenere), Betrachtungszeiträume sowie geografisch bedingte Unterschiede. Im Rahmen einer Messnetzabfrage zur atmosphärischen Deposition von PAKs in Deutschland (Zeitraum Juli 2007 bis Januar 2008) wurden aktuelle Daten des Luftmessnetzes des Umweltbundesamtes sowie der Landesmessnetzzentralen aller 16 Bundesländer erhoben (Abb. 1), wobei jedoch nicht alle Bundesländer Daten zur Verfügung stellen konnten (Götz 2008).

Bisher dominieren Verfahren zur Erfassung von PAKs im Zusammenhang mit der allgemeinen Staubdeposition sowie der PM10-Konzentration in der Umgebungsluft. Werden bei der Staubdeposition nur einzelne Phasen (Trocken- oder Nassdepositionsflüsse) berücksichtigt, ist eine vollständige Erfassung von PAKs jedoch nicht gewährleistet. Bei Messungen von PAKs in der PM10-Fraktion ist von einem Einfluss durch die Wahl der Messstandorte (oftmals Belastungsschwerpunkte) sowie durch die notwendige Umrechnung in Depositionsraten auszugehen. Seit dem Jahr 2002 existiert nach DIN 19739 (Trichter-Adsorber-Sammler) ein spezifisch für PAKs optimiertes Verfahren zur Depositions- 


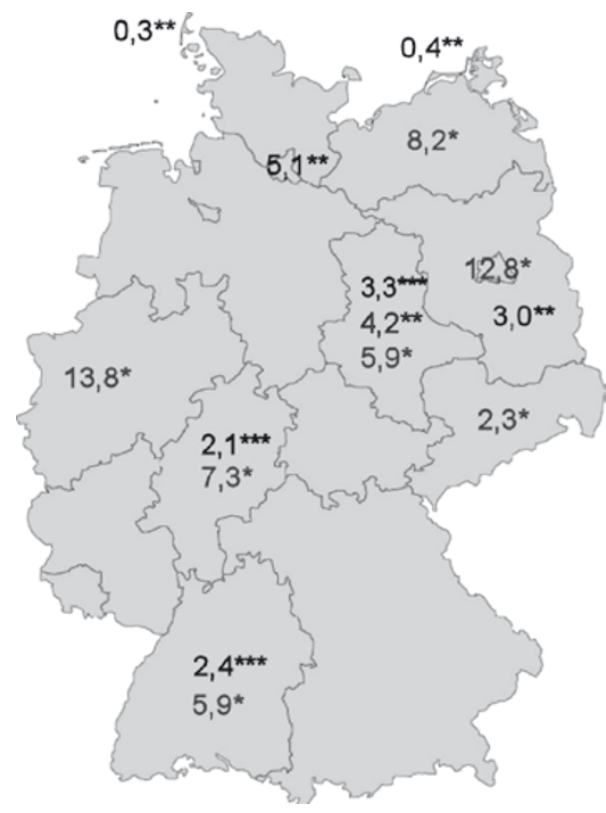

Abb. 1 Mittlere PAK-Depositionsraten in den Bundesländern in g/(ha a), Götz (2008), Messung: * PM10 Umgebungsluft, ** (Staub) Depositionsmessung, ***nach DIN 19739

messung, welches aktuell jedoch nur in wenigen Messnetzen eingesetzt wird. Für Deutschland errechnet sich anhand der verfügbaren Mittelwerte der einzelnen Bundesländer eine mittlere Deposition von 6,8g/(ha a) $\Sigma$ EPA-PAK16. Für eine Einordnung dieses Wertes können Daten vergleichbarer internationaler Studien herangezogen werden. So wurden zum Beispiel von Moon et al. (2006) in Südkorea für die Jahre 2002 bis 2004 mittlere Höchstwerte der Deposition von $\Sigma$ EPA-PAK16 von $6,1 \mathrm{~g} /($ ha a) in urbanen Gebieten und $4,6 \mathrm{~g} /($ ha a) in suburbanen Gebieten bestimmt. Moon zitiert andere internationale Studien wie Ollivon et al. (2002), der für Paris (PAK14) Depositionen von 7,3 g/(ha a) bestimmte, oder Rossini et al. (2005), der Werte (PAK16) von ca. 0,07 bis zu 7,3 g/(ha a) in Venedig ermittelte. Die Vergleichswerte bei Moon et al. (2006) deuten darauf hin, dass in den Jahren nach 2000 ein Mittelwert von 6,8g/(ha a) für Deutschland als Worst-case-Szenario betrachtet werden kann. Die ausgewerteten Messwerte wurden häufig an Belastungsschwerpunkten gemessen, so in den dicht besiedelten industriellen und urbanen Zentren (z. B. Berlin, Ruhrgebiet) mit Größenordnungen von ca. 10 bis $15 \mathrm{~g} /($ ha a). Dagegen weisen die aktuell vorhandenen Daten für weniger belastete (bezogen auf die Fläche) aber bedeutende ländliche Gebiete eine Deposition von ca. 1 bis $3 \mathrm{~g} /($ ha a) aus.

Hierbei spielt der grenzüberschreitende Stofftransfer eine wichtige Rolle. So können regional in Deutschland erhöhte Depositionen aufgrund der typischen atmosphärischen Strömungsverhältnisse mit der Emissionssituation in Nachbarländern in Verbindung gebracht werden (z. B. Sachsen: östliche europäische Nachbarländer mit starkem Einsatz von Kohlebrennstoffen; Nordrhein-Westfalen: industrielle Emissionen in den Benelux-Staaten). Weiterhin führt der Einfluss der Heizperiode zu deutlichen Unterschieden bei der mittleren atmosphärischen Deposition um ca. den Faktor 2 zwischen Messungen im Sommer und im Winter (Umweltbeobachtung 2007).

\subsubsection{Abschwemmung von unbefestigten Flächen}

Bei der Quantifizierung der gelösten Stoffemissionen durch Oberflächenabfluss von unbefestigten Flächen ist als wesentliche Komponente der abfließende Niederschlag zu berücksichtigen (LfUG 2008). Die $\Sigma$ EPA-PAK16-Konzentration in Niederschlagswässern in Deutschland beträgt 0,3 bis $9,4 \mu \mathrm{g} / \mathrm{L}$ und ist somit durch eine große Schwankungsbreite gekennzeichnet (Welker 2004). Im Rahmen des Abwasserbeseitigungsplans Berlin (2001) wurden Werte von 0,25 bis $1 \mu \mathrm{g} / \mathrm{L}$ gemessen. Neben potenziellen Emissionsquellen ist von einem wesentlichen Einfluss durch die jeweils regional dominierenden Niederschlagsverhältnisse und somit dem Ort der Probenahme auszugehen. Da im Fall des Oberflächenabflusses von unbefestigten Flächen bei PAKs zudem mit Wechselwirkungen mit dem Bodensubstrat zu rechnen ist (Sorptionsprozesse), wird für die nationale Abschätzung der niedrigste erfasste Wert von $0,25 \mu \mathrm{g} / \mathrm{L}$ eingesetzt.

\subsubsection{Erosion}

Das Vorkommen von PAKs in Böden kann vor allem als Folge des atmosphärischen Eintrags betrachtet werden (Hellmann 2004). Dabei übertrifft der „trockene“ Anteil in Form der Staubdeposition den „nassen“ Anteil deutlich. Zusätzliche PAK-Einträge sind auf den Einsatz von Klärschlämmen und anderen Düngern in der Landwirtschaft zurückzuführen (UBA 2007b). Von einer signifikanten Stoffverlagerung in den Untergrund unterhalb der Bearbeitungstiefe ist jedoch in der Regel nicht auszugehen (Hellmann 2004). Infolge des bevorzugten Transportes von feinen Bodenpartikeln kommt es während des Erosionsprozesses zu einer Anreicherung feiner Kornklassen im abgetragenen Sediment (Fuchs et al. 2002). Die Anreicherung eines Stoffes im Erosionsgut wird durch das Anreicherungsverhältnis im Vergleich zum Ausgangsgehalt des Stoffes im Ackeroberboden beschrieben. Die PAK-Gehalte in Oberböden (LABO 2003) weisen in den einzelnen Bundesländern deutliche Unterschiede auf, wobei jedoch nicht für alle Länder Daten zur Verfügung stehen. Als wesentliche Ursachen für Abweichungen sind neben den regionalen Unterschieden variierende Bezugszeiträume, die Anzahl der untersuchten PAK-Verbindungen sowie die Differenzierung nach Landnutzung zu nennen. Liegt eine differenzierte Angabe verschiedener Typen von Ackerböden vor, wird in Abhängigkeit von der Anzahl der 
jeweils verfügbaren Proben ein gewichteter Mittelwert gebildet. Entsprechend Kollotzek et al. (1996) wird ein Verhältnis von $\Sigma$ EPA-PAK16 $=$ Borneff PAK6 $\times 2,3$ angenommen. Sind keine Daten für ein Bundesland verfügbar, wird der Mittelwert von $0,38 \mathrm{mg} / \mathrm{kg}$ für Deutschland (EPAPAK16) verwendet, welcher aus den Werten der anderen Bundesländer gebildet wird. Scheffer et al. (2002) nennt für Deutschland einen natürlichen Hintergrundwert von 1 bis $10 \mu \mathrm{g} / \mathrm{kg}$ sowie aktuelle mittlere Gehalte von $0,2 \mathrm{mg} / \mathrm{kg}$ auf Ackerflächen, $0,4 \mathrm{mg} / \mathrm{kg}$ in Waldböden und $1,1 \mathrm{mg} / \mathrm{kg}$ in urbanen Gebieten.

\subsubsection{Grundwasser, Drainagewasser bzw Sickerwasser}

Die Grundwasserdatenbank des Umweltbundesamtes mit 800 Messstellen in Deutschland umfasst zum Abfragezeitraum im Sommer 2008 PAK-Daten für insgesamt sieben Messstellen aus zwei Bundesländern, wobei alle Werte unterhalb der jeweiligen Bestimmungsgrenzen liegen (UBA 2008c). Ergänzend wurde daher eine Abfrage der zuständigen Landesmessnetzzentralen aller 16 Bundesländer durchgeführt, wobei jedoch ebenfalls nur wenige Bundesländer Messwerte oberhalb der Bestimmungsgrenze zur Verfügung stellen konnten. Signifikante PAK-Grundwasserkontaminationen unter nicht extrem belasteten Standorten sind demnach aufgrund der geringen Wasserlöslichkeit sowie weitreichender Sorption an Humusstoffe und Tonminerale in Oberflächennähe nicht zu erwarten. Aktuelle Messungen mit niedrigeren Bestimmungsgrenzen ergeben einen Mittelwert von $0,02 \mu \mathrm{g} / \mathrm{L}$ EPA-PAK16 (Berlin 2008), der für eine erste großräumige Betrachtung eingesetzt wird. Hierbei ist zu beachten, dass bei positiven Befunden vor allem Naphthalin, Phenanthren und Fluoranthen nachgewiesen werden können, während die verbleibenden Verbindungen der TVO PAK6 oder EPA-PAK16 Summenparameter oftmals nicht oder nur in geringem Umfang vorgefunden werden. Nach Messungen durch Schramm (2008) in Trinkwasser (Alpenvorland) ist zudem in anthropogen gering beeinflussten Gebieten von Konzentrationen auszugehen, die nochmals deutlich unterhalb der Bestimmungsgrenzen der allgemeinen Grundwasserüberwachung liegen. Da jedoch nur sehr wenige Messwerte mit sensitiveren Analysemethoden vorliegen, ist es bisher nicht möglich, für eine nationale $\mathrm{Ab}$ schätzung eine mittlere PAK-Konzentration im Grundwasser abzuleiten.

Für PAKs in Drainagewasser liegen ebenfalls nur in sehr geringem Umfang Daten zu Konzentrationen vor. Der Oberboden wird aufgrund der Bindung an organische Substanz und Tonminerale sowie der geringen Wasserlöslichkeit der PAK-Kongenere als die Hauptsenke für Stoffeinträge betrachtet (Scheffer et al. 2002). Wie im Fall des Grundwassers wird daher eine mittlere $\Sigma$ EPA-PAK16 Hintergrundkonzentration von $0,02 \mu \mathrm{g} / \mathrm{L}$ angenommen.

\subsubsection{Urbane Flächen}

PAK-Konzentrationen im Abfluss urbaner Flächen zeigen eine sehr hohe Schwankungsbreite, wobei neben der Analytik (Anzahl untersuchter PAK-Verbindungen) als wesentliche weitere Einflussfaktoren vor allem Ort (z.B. Ursprungsland, Stadt, Verkehrswege) und Zeitpunkt (z. B. Jahr, Jahreszeit) der Probenahme zu nennen sind. Im Rahmen einer Datenerhebung für Straßenabläufe wurde eine Konzentration von im Mittel 3,5 $\mu \mathrm{g} / \mathrm{L}$ EPA-PAK16 bestimmt (IWG 2008). Mithilfe des Bilanzierungsmodells MONERIS, welches am Leibnitz-Institut für Gewässerökologie und Binnenfischerei von Dr. Behrendt ursprünglich für Nährstoffbilanzen in Flussgebieten entwickelt wurde, konnte aus dieser Konzentration ein mittleres Oberflächenpotenzial (Stoffeintrag pro Fläche und Zeiteinheit) von $10,9 \mathrm{~g} /$ (ha a) zurückgerechnet werden. Als Vergleichsbasis können die Einzelbeiträge zum Oberflächenpotenzial urbaner Flächen auf Grundlage von PAK-Konzentrationen in Reifen- und Bremsenabrieb (UBA 2008a), Abriebsmengen (Hillenbrand et al. 2005) sowie Angaben zu Verkehrsflächen in Deutschland (Destatis 2008) errechnet werden. Das so ermittelte Gesamtoberflächenpotenzial für Verkehrsflächen in Deutschland aus „atmosphärischer Deposition“ ((6,8g/(ha a)), vgl. oben) und abriebbedingten PAK-Einträgen $(5,1 \mathrm{~g} /(\mathrm{ha}$ a) $)$ beträgt im Mittel ca. 11,9g/(ha a) (Fuchs et al. 2008). Somit ist eine große Übereinstimmung mit dem aus Verkehrsflächenabflüssen zurückgerechneten Oberflächenpotenzial von $10,9 \mathrm{~g} /$ (ha a) festzustellen. Hierbei ist zu beachten, dass jeweils Verkehrsflächen berücksichtigt werden, die jedoch nur einen Anteil von ca. 38\% an der Flächenkategorie „Siedlungs- und Verkehrsflächen“ besitzen (Destatis 2008). 72\% der Fläche entfallen dagegen auf die vorherrschend durch die atmosphärische Deposition beeinflussten Nutzungsarten „Gebäude- und Freiflächen“, „Betriebsfläche ohne Abbauland“, „Erholungsfläche“ sowie „Friedhof“ (Destatis 2008). Für die weitergehende Berechnung für den Eintragspfad „urbane Flächen" wird daher ein anhand der Flächenanteile gewichtetes Mittel von $9 \mathrm{~g}$ pro Hektar und Jahr verwendet.

Zukünftig ist mit einem deutlichen Rückgang der abriebbedingten Emissionen aus dem Verkehrsbereich zu rechnen. Auf europäischer Ebene wurden durch RL 2005/69/EG Beschränkungsmaßnahmen für PAKs in Reifen im Straßenverkehr erlassen. Als Folge der Umsetzung der Richtlinie 2005/69/EG ist bereits aktuell bei zahlreichen neuen Reifenmodellen nur noch eine geringe PAK-Belastung feststellbar (ADAC 2008). Bis zum Jahr 2012 wird eine Reduktion von PAK-Emissionen aus Reifen um insgesamt mehr als 95\% erwartet (UMWELT 2006).

\subsubsection{Binnenschifffahrt und Sportboote}

Nachdem teerölhaltige Schiffsanstriche aufgrund der inzwischen vorgeschriebenen Überholungszeiträume keine we- 
sentliche Bedeutung mehr besitzen (Gandrass und Salomons 2001), erfolgen PAK-Emissionen der Binnenschifffahrt vor allem durch Abgase in die Luft (MAN Diesel 2008; ZKR 2008) und tragen damit indirekt über den oben geschilderten Pfad ,atmosphärische Deposition“ zu Gewässerbelastungen bei. Außenbordmotoren von Sportbooten verursachen dagegen bedeutende direkte Gewässereinträge, da der Abgasstrom unmittelbar ins Schraubenwasser eingebracht wird (Horn et al. 2005). Horn et al. ermittelten Gewässeremissionen von 48 bis $216 \mathrm{mg}$ PAK16 bei einer Betriebszeit von einer halben Stunde. Mit dem niedrigeren Wert und ca. 200.000 Booten mit Außenbordmotoren in Deutschland sowie einer unterstellten mittleren Nutzung von ca. $60 \mathrm{~h}$ im Jahr errechnet sich ein Gesamteintrag von ca. 1,2t im Jahr. Aufgrund der wenigen vorliegenden Messungen ist dieser Wert allerdings mit starken Unsicherheiten behaftet (Fuchs et al. 2008). Für die Verteilung der Einträge durch Außenbordmotoren auf die MONERIS-Modellgebiete wurden nur die schiffbaren Abschnitte der Wassereinzugsgebiete erster Ordnung in ihrer Wasserfläche berücksichtigt. Dies ist eine erste methodische Näherung; in Folgeprojekten wären Karten der Wasserstrassen mit den Karten der Modellflussgebiete zu verschneiden, um die schiffbaren Flächen zu erhalten.

In Kooperation mit der Bundesanstalt für Wasserbau (BAW) in Karlsruhe wurde zusätzlich versucht, die Emissionen aus alten, PAK-haltigen Korrosionsschutzanstrichen im Stahlwasserbau abzuschätzen. In der Vergangenheit kamen Steinkohlenteerpechhaltige Farben (STKP) zum Einsatz, die in der Folge von Teerepoxiden (TE) und dann von Epoxid-Teerersatzstoffen (EP-TE) abgelöst wurden. Aus der BAW Datenbank ergibt sich über die Parameter Durchrostung, Bauteilfläche, spez. Gewicht und Schichtdicke der Farben sowie einer Lebensdauer von 20 Jahren ein Verlust durch abblätternde Farbe von rund $45 \mathrm{~kg}$ pro Jahr (Fuchs et al. 2008). Zusätzlich kann eine Diffusion von PAKs aus der Farbschicht in die Wasserphase erfolgen, die jedoch schwierig abzuschätzen ist, da entsprechende Analysen fehlen. Geht man in einer „Worst-case-Abschätzung“ davon aus, dass alle Bauteile in der Datenbank mit Steinkohlenteerpech behandelt sind, errechnet sich ein Verlust von $0,35 \mathrm{t}$ pro Jahr aus der Beschichtung (Fuchs et al. 2008). Insgesamt beläuft sich der abgeschätzte Eintrag aus dem Bereich Korrosionsschutz für Stahlwasserbauten für das Jahr 2004 somit auf ca. $400 \mathrm{~kg}$. Diese Menge ist aufgrund der abnehmenden Nutzung von PAK-haltigen Farben rückläufig.

\subsubsection{Einträge durch sonstige Produkte}

Obwohl der Einsatz von PAKs in verschiedenen Produkten wie Wurfscheiben, teerhaltigen Klebern, Teerölfarben zum Korrosionsschutz, als Weichmacher in Gummiprodukten, als Wirkstoff in Mottenkugeln und in anderen Spezialprodukten stark eingeschränkt worden ist, erfolgen aus belaste- ten Altprodukten, aber auch aus verschiedensten neuen Produkten (z. B. Baumaterialien, Werkzeugen und Bekleidung) nach wie vor Emissionen in die Umwelt und die Gewässer (TEST 2006). In Bezug auf die Größenordnung der resultierenden Einträge besteht weiterhin großer Forschungsbedarf (Fuchs et al. 2008).

\section{Vergleich der berechneten PAK-Emissionen mit Gewässerfrachten an Gütepegeln}

\subsection{Bewertung der Datengrundlage}

Die Beschreibung für die einzelnen Eintragspfade zeigt die bestehenden, zum Teil sehr großen Unsicherheiten bei der Quantifizierung der in Gewässer eingetragenen Frachten. Dies betrifft insbesondere den Bereich des Grundwasserzuflusses, für den nur wenige Messdaten oberhalb der Bestimmungsgrenze vorliegen sowie den Erosionspfad, für den es bislang keine Untersuchungen zur möglichen Anreicherung von PAKs aufgrund des bevorzugten Transports von feinen Bodenpartikeln gibt. Für den Bereich der urbanen Flächen ist zu berücksichtigen, dass die verfügbaren Daten eine sehr große Schwankungsbreite aufweisen und Messungen zudem oftmals nur für reine Verkehrsflächen vorliegen. Zusätzlich wird die Vergleichbarkeit von Untersuchungen durch die unterschiedliche Anzahl der untersuchten PAK-Verbindungen stark eingeschränkt. Die Verhältnisse der Stoffmengen können räumlich und zeitlich aufgrund der Abhängigkeit von den Emissionsquellen schwanken, eine Umrechnung von Einzelsubstanzen auf die Summe PAK ist mit zusätzlichen Fehlern behaftet. Obwohl zum Teil noch weiterer Forschungsbedarf besteht, soll hier eine erste Bewertung der Relevanz der einzelnen Eintragspfade erfolgen (Abb. 2). Anhand der sich ergebenden Relevanzverteilung wird deut-

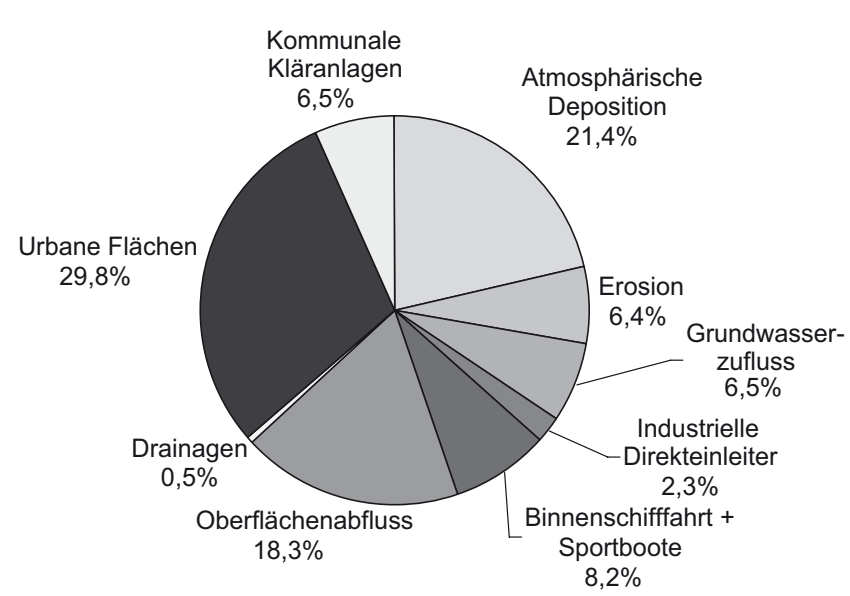

Abb. 2 Relative Relevanz der Eintragspfade/PAK-Gewässereinträge 2005 (Fraunhofer ISI) 
lich, dass von einem dominierenden Einfluss diffuser atmosphärischer Einträge am Gesamteintrag von PAKs in Gewässer auszugehen ist. Neben der direkten Deposition auf die Gewässeroberflächen (,atmosphärische Deposition“) trägt diese Quelle auch indirekt wesentlich zu den Gewässereinträgen von urbanen Flächen sowie den Einträgen infolge von Erosion und Oberflächenabfluss bei. Darüber hinaus ist auch bei den Stoffeinträgen aus Kläranlagenabläufen eine starke Beeinflussung durch die atmosphärische Deposition auszugehen. Der Eintrag erfolgt dabei über Flächen im Einzugsgebiet der Abwasserbehandlungsanlagen, die an ein Mischsystem angeschlossen sind (LfUG 2008).

4.2 Vergleich von berechneten und gemessenen PAK-Flussfrachten

Auf Grundlage der erhobenen Daten und einer Bewertung der flussinternen Retention von PAKs kann zusätzlich mit MONERIS ein Vergleich von berechneten und gemessenen Flussfrachten sowie eine Plausibilitätsprüfung für das Ergebnis erfolgen. Zur Berechnung der von der spezifischen Abflussspende $q$ abhängigen Retention $R_{\mathrm{L}}$ wurde das Retentionsmodell nach Behrendt benutzt (Vink und Behrendt 2002): $R_{\mathrm{L}}=a \cdot q^{\mathrm{b}}$, die gewählten Faktoren waren $a=0,07$ und $b=1,3$ (Fuchs et al. 2002).

Abbildung 3 stellt den Zusammenhang zwischen berechneter Fracht (Differenz: Emission - Retention) und Gewässerfracht dar (ermittelt aus 88 deutschen Pegeln, für die EPA PAK16-Daten aus den Jahren 2003 bis 2005 vorlagen). Ergänzend wird die 50\% Abweichung von der 1:1-Linie als punktierte Linie abgebildet. Tendenziell sind hierbei an
Pegelmessstellen mit großen PAK-Frachten deutlich geringere Abweichungen von der 1:1-Linie festzustellen, als an Standorten mit geringeren Frachten. Vor allem bei den großen Flussgebieten, wie z. B. Rhein und Elbe, können die PAK-Emissionen weitestgehend mit einer Abweichung von weniger als $50 \%$ ermittelt werden (Fuchs et al. 2008). Bei geringen Frachten und kleinen Flussgebieten liegt die berechnete Fracht in der Regel deutlich über der gemessenen. Als mögliche Ursache sind die PAK-Messdaten von Pegelmessstellen zu nennen, die nicht für alle Flussgebiete in gleicher Qualität (zeitliche Auflösung, Anzahl der erfassten PAK-Vertreter) und Quantität (Dichte des Messnetzes) zur Verfügung stehen. Sind die berechneten Einträge signifikant größer als die nachweisbaren Gewässerfrachten, kann zudem oftmals ein Zusammenhang mit der Lage von Gütemessstellen unterhalb von Seen oder Talsperren hergestellt werden, die hinsichtlich der Retention besondere Einflüsse ausüben können (Fuchs et al. 2008). Untersuchungen unterstreichen aber auch den Einfluss von Geosorbentien wie Kohlen auf die Sorption und Desorption von PAK (Pies 2002).

\subsection{Ausblick und Lösungsansätze}

PAK-Minderungsmaßnahmen zeigen bei vielen Quellen bereits deutliche Wirkung. Aufgrund komplexer Stofftransferprozesse besitzen die atmosphärischen PAK-Einträge jedoch weiterhin eine große Relevanz für die Belastungen der Oberflächengewässer (Fuchs et al. 2008). Entsprechend den Angaben des Schadstofffreisetzungs- und -verbringungsregisters (PRTR 2007) für den Zeitraum 1990 bis 2005 besitzt der Hausbrand in Deutschland mit durchschnittlich $>80 \%$
Abb. 3 Vergleich zwischen berechneten Frachten mit Gewässerfrachten an Gütepegeln (Fraunhofer ISI)

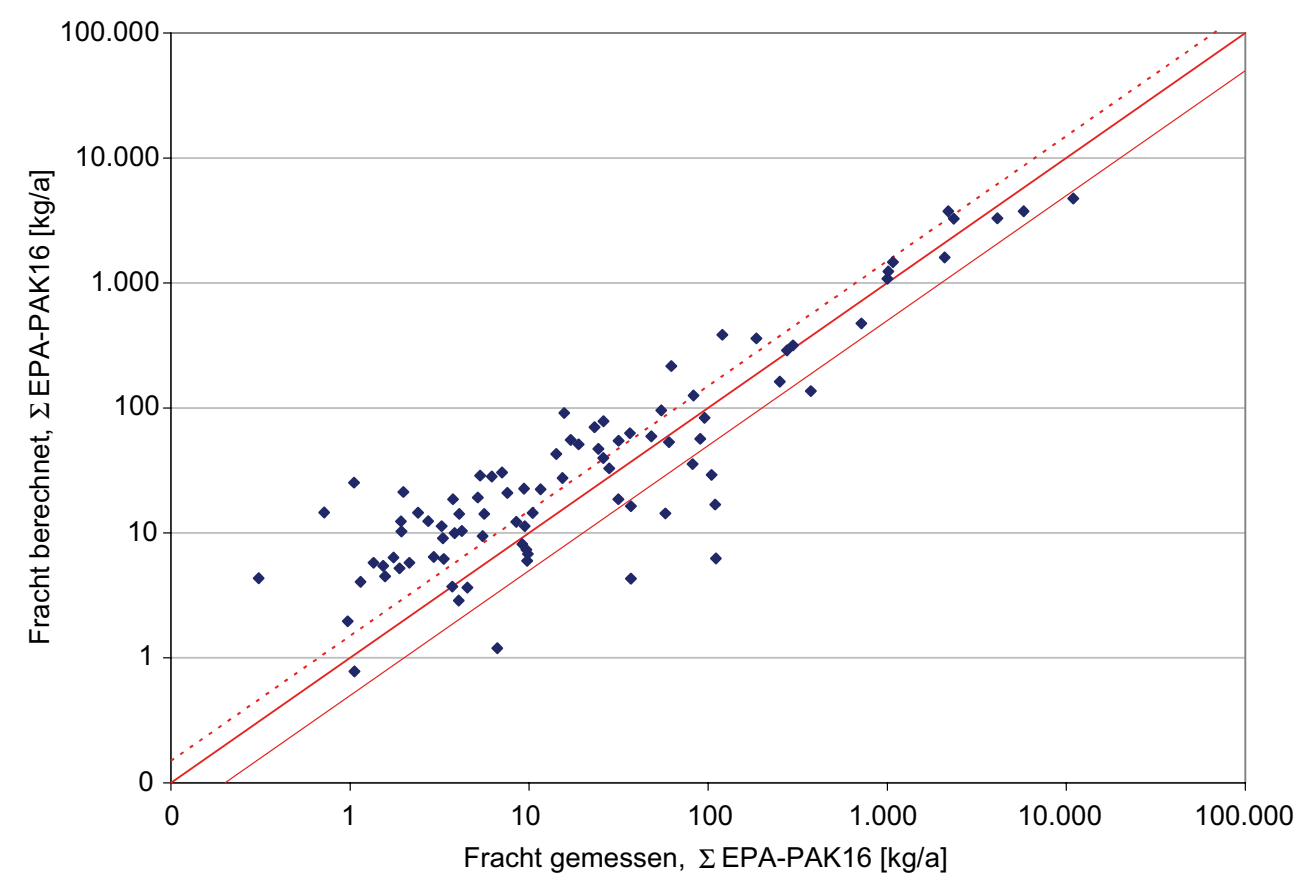


Abb. 4 Einfluss der Emissionen des Hausbrandes auf EPAPAK16-Gewässereinträge 2025 (Fraunhofer ISI)

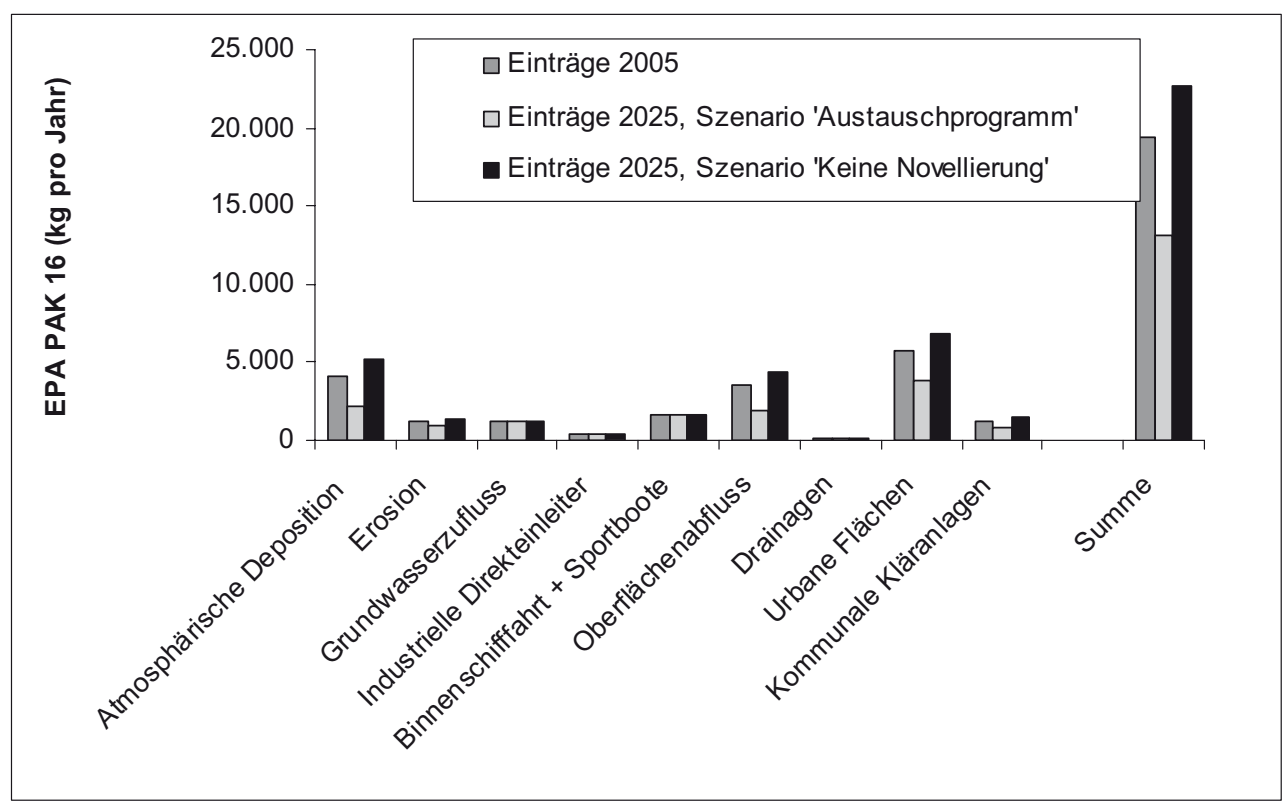

den größten Anteil an den erfassten diffusen PAK-Gesamtemissionen in die Atmosphäre. Innerhalb des Bereichs Hausbrand ist dabei der Anteil der Holzbrennstoffe in den vergangenen Jahren stark gestiegen und kompensiert damit den Rückgang bei Kohlebrennstoffen (UBA 2007d). Aufgrund der voraussichtlichen Preisentwicklung bei Öl und Gas und der Förderung $\mathrm{CO}_{2}$-neutraler Brennstoffe ist in $\mathrm{Zu}$ kunft von einer weiteren starken Zunahme im Bereich der Holzfeuerungsanlagen auszugehen.

Eine erste grobe Wirkungsabschätzung der weiteren Emissionsentwicklung bei Kleinfeuerungsanlagen kann auf der Grundlage von Szenarien des Umweltbundesamtes im Zusammenhang mit der geplanten Novellierung der 1. BimSchV (UBA 2007d) und der Annahme einer zu Feinstäuben proportionalen Entwicklung der PAK-Emissionen erfolgen (Abb. 4). Im Szenario „keine Novellierung“ wird aufgrund der oben genannten Gründe von einer weiteren starken Zunahme im Bereich der Holzfeuerungsanlagen bis zum Jahr 2025 ausgegangen. Dadurch ergibt sich gegenüber dem Jahr 2005 eine Zunahme um ca. $3000 \mathrm{~kg}$ EPA PAK16. Im Szenario „Austauschprogramm“ werden strenge Emissionsanforderungen an neue, aber auch an im Betrieb befindliche Heizanlagen gestellt, so dass ab sofort eine Reduktion der Feinstaubemissionen stattfindet. Die Emissionen in Gewässer würden sich dadurch im Jahr 2025 gegenüber 2005 um mehr als $6000 \mathrm{~kg}$ verringern. Die Berechnungen für die von der atmosphärischen Deposition direkt und indirekt beeinflussten Eintragspfade verdeutlichen, dass die Durchführung oder Unterlassung von Maßnahmen im Bereich des Hausbrandes entscheidend für die weitere Entwicklung von PAK-Einträgen in Gewässer sind.

\section{Schlussfolgerungen}

Zur weiteren Reduktion von Überschreitungen von Umweltqualitätsnormen in Gewässern besteht dringender Handlungsbedarf. Hierfür sind zusätzliche Emissionsminderungsmaßnahmen für PAK in unterschiedlichen Bereichen notwendig, für deren Priorisierung die vorliegende Arbeit erste Ansatzpunkte liefert. Durch die Begrenzung von PAK-Emissionen im Straßenverkehr, wie etwa infolge des Einsatzes von Partikelfiltern bei Dieselfahrzeugen sowie durch die Begrenzung von PAKs in Reifen, wird ein deutlicher Rückgang verkehrsbedingter Einträge erwartet. Daraus ergibt sich für Gewässer eine weiter zunehmende Relevanz der verbleibenden Quellen, wie etwa den diffusen atmosphärischen PAK-Einträgen (Fuchs et al. 2008).

Danksagung Die Arbeiten erfolgten im Wesentlichen im Rahmen der von Seiten des Umweltbundesamtes geförderten Forschungsvorhaben „Prioritäre Stoffe der Wasserrahmenrichtlinie - Europäische Regelung und nationales Maßnahmenprogramm“ und „Modell-basierte Quantifizierung und Internet-basierte Visualisierung der Einträge prioritärer Stoffe in die Fließgewässer Deutschlands“.

\section{Literatur}

ADAC (2008) PAK: Schadstoffen auf der Spur ADAC-PAK-Test. www.adac.de/tests/reifentests/pak/default.asp?componentid= 163988\&SOURCEPAGEID $=8979 \&$ TL $=2$ (abgerufen im Juni 2008)

Berlin (2001) Abwasserbeseitigungsplan, http://www.berlin.de/sen/ umwelt/wasser/download/AB-Plan4.pdf, Senatsverwaltung Stadtentwicklung

Berlin (2008) http://www.berlin.de/sen/umwelt/wasser/hydrogeo/de/ broschuere/2.4-Grundwasserbeschaffenheit.pdf (abgerufen im Juli 2008) 
Blumer M (1976) Polycyclic aromatic compounds in nature. Sci Am 234(3):34-45

BMU (2006) Bundesministerium für Umwelt, Naturschutz und Reaktorsicherheit, „Wasserwirtschaft in Deutschland“

BMU (2007) Abfallwirtschaft Klärschlammverwertung in der Landwirtschaft. Deutscher Klärschlamm-Bericht, EU-Kommission. http://www.bmu.de/abfallwirtschaft/doc/40230.php (Stand: Oktober 2007)

Destatis (2004) Öffentliche Wasserversorgung und Abwasserbeseitigung 2004. Statistisches Bundesamt, Fachserie 19/Reihe 2.1: Umwelt Öffentliche Wasserversorgung und Abwasserbeseitigung, 10. Abwasserbehandlungsanlagen 10.1

Destatis (2008) Nutzung von Fläche und Raum Siedlungs- und Verkehrsfläche nach Art der tatsächlichen Nutzung. http://www.destatis.de/jetspeed/portal/cms/Sites/destatis/Internet/DE/Content/ Statistiken/Umwelt/UmweltoekonomischeGesamtrechnungen/ Flaechennutzung/Tabellen/Content75/SiedlungsVerkehrsflaeche Nutzung,templateId=renderPrint.psml (abgerufen im Juli 2008)

EPER (2007) Ergebnisse der Berichterstattungen zum Europäischen Schadstoffemissionsregister EPER-1 und EPER-2 in Deutschland. http://www.home.eper.de/startseite/eper_daten/ergebnisse/ Auswertung\%20EPER_Bericht1+2.pdf (Stand: Juli 2007)

EUWID (2008) EUWID Wasser und Abwasser, Wa Nr. 30. http:// www.euwid-wasser.de (abgerufen am: 22. Juli 2008)

Fuchs S, Scherer U, Hillenbrand T, Marscheider-Weidemann F, Behrendt H, Opitz D (2002) Schwermetalleinträge in die Oberflächengewässer Deutschlands. Texte 54/02, Umweltbundesamt, Berlin, $166 \mathrm{~S}$

Fuchs S et al. (2008) Modell-basierte Quantifizierung und Internet-basierte Visualisierung der Einträge prioritärer Stoffe in die Flussgebiete Deutschlands. Endbericht zum UBA F+E Vorhaben FKZ 20424218 (in Vorbereitung)

Gandrass J, Salomons W (2001) Dredged Material in the Port of Rotterdam - Interface between Rhine Catchment Area and North Sea. Project for the Port of Rotterdam. GKSS, Geesthacht

Gocht T, Grathwohl P (2004) Polyzyklische aromatische Kohlenwasserstoffe aus diffusen Quellen: Atmosphärische Deposition und Anreicherung in Böden des ländlichen Raums Umweltwiss Schadst Forsch 16(4):245-254

Götz T (2008) Einträge von prioritären Stoffen in Gewässer Untersuchungen am Beispiel von PAK und Blei, Institut für Geographie und Geoökologie (IFGG I) der Universität Karlsruhe (TH) in Kooperation mit dem Fraunhofer Institut für System- und Innovationsforschung (ISI), Diplomarbeit. http://publica.fraunhofer.de/ starweb/servlet.starweb?path=urn.web\&search=urn:nbn:de:0011n-776189

Hellmann H (2004) Vorschlag zur Auswertung von PAK - Kontaminationen. Umweltwiss Schadst Forsch 15(3):175-184

Henzler R (2004) Quantifizierung und Modellierung der PAK-Elution aus verfestigten und unverfestigten Abfallmaterialien. Dissertation, Tübingen

Hillenbrand T, Böhm E, Fuchs S, Scherer (2005) Einträge von Kupfer, Zink und Blei in Gewässer und Böden - Analyse der Emissionspfade und möglicher Emissionsminderungsmaßnahmen. Texte 19/05, Umweltbundesamt, Dessau

Hillenbrand T, Marscheider-Weidemann F (2006) Datenblatt Nr. 28: PAK, Forschungsvorhaben „Emissionsminderung für prioritäre und prioritäre gefährliche Stoffe der Wasserrahmenrichtlinie“, Anthracen, Naphthalin und Fluoranthen. http://www.umweltdaten.de/wasser/themen/stoffhaushalt/pak_anthracen_naphthalin_und_fluoranthen.pdf

Hillenbrand T, Marscheider-Weidemann F, Götz T (2008) Datenbasis Prioritäre Stoffe. Relevanz in Deutschland sowie weitergehende Untersuchungen am Beispiel der PAK. In: Fuchs S. et al. (2008) Stoffströme in Flussgebieten - Von der Bilanzierung zur Bewirtschaftung
Ivashechkin P (2005) Literaturauswertung zum Vorkommen gefährlicher Stoffe im Abwasser und in Gewässern. Projekt AZ IV 9- 042 059 für das MUNLV, S46-49

IWG (2008) Institut für Wasser und Gewässerentwicklung, Bereich Siedlungswasserwirtschaft und Wassergütewirtschaft. Scherer U: Datensammlung zu gemessenen PAK-Konzentrationen im Trennkanal im Mittel ab 1995

Kollotzek D et al. (1996) Technische, analytische, organisatorische und rechtliche Maßnahmen zur Verminderung der Klärschlammbelastung mit relevanten organischen Schadstoffen. FuE-Projekt 10350123 des Umweltbundesamtes

LABO (2003) Bund-Länder-Arbeitsgemeinschaft Bodenschutz, LABO, Hintergrundwerte für anorganische und organische Stoffe in Böden, 3. überarb. und erg. Aufl. Anhang: Tabellen der Hintergrundwerte für Böden

Lehmann M, Vietoris U (2006) Prioritäre Stoffe - Ergebnisse Länderabfrage 2005. Vortrag im Rahmen des Workshops „Emissionsminderungsmaßnahmen für prioritäre Stoffe der Wasserrahmenrichtlinie - Bestandsaufnahme und Handlungsoptionen“, 30. Mai 2006. http://www.umweltbundesamt.de/wasser/themen/ stoffhaushalt/ws-prio-stoffe.htm

LFUG (2008) Ermittlung und Quantifizierung von Quellen für ausgewählte Schadstoffbelastungen in sächsischen Flusseinzugsgebieten und Ableitung von Maßnahmen unter Einbeziehung von Kosteneffizienz-Aspekten, Teil: Ermittlung zur Belastung mit PAK. Sächsisches Landesamt für Umwelt und Geologie, Leipzig

MAN Diesel (2008) Persönliche Mitteilung

Moon H-B, Kannan K, Lee S-J, Ok G (2006) Atmospheric Deposition of Polycyclic Aromatic Hydrocarbons in an Urban and a Suburban Area of Korea from 2002 to 2004. Arch. Environ. Contam. Toxicol. 51:494-502

Ollivon D, Blanchoud H, Motelay-Massei A, Garban B (2002) Atmospheric deposition of PAHs to an urban site, Paris, France. Atmospheric Environ (36):2891-2900

PRTR (2007) Pollutant Release and Transfer Register, Datengrundlage für PAK. http://www.prtr.de/ (abgerufen im Dezember 2007)

Rossini P, Guerzoni S, Molinaroli E, Rampazzo G, De Lazzari A, Zancanaro A (2005) Atmospheric bulk deposition to the lagoon of Venice. Part I. Fluxes of metals, nutrients and organic contaminants. Environment international 31(7):959-974

Schramm K-W (2008) Prüfbericht: „Bestimmung von polyzyklischen aromatischen Kohlenwasserstoffen" Deutsches Forschungszentrum für Gesundheit und Umwelt, Helmholtz Zentrum München

TEST (2006) Gift in Werkzeugen. Stiftung Warentest. http://www. stiftung-warentest.de/online/haus_garten/meldung/1365869/ 1365869.html

UBA (2007b) Begrenzung von Schadstoffeinträgen bei Bewirtschaftungsmaßnahmen in der Landwirtschaft bei Düngung und Abfallverwertung, UBA Texte 30/07. www.umweltdaten.de/publikationen/fpdf-1/3295.pdf

UBA (2007c) Hintergrundpapier Feinstaub aus Kamin und Holzofen. http://www.umweltbundesamt.de/uba-info-presse/hintergrund/ holzfeuerung.pdf,

UBA (2007d) Hintergrund, Novelle der Kleinfeuerungsverordnung. www.bmu.de/files/pdfs/allgemein/application/pdf/bimschv1_hg novelle.pdf

UBA (2008a) Nationaler Durchführungsplan unter dem Stockholmer Abkommen zu persistenten organischen Schadstoffen (POPs), Texte $01 / 08$

UBA (2008b) Berichterstattung 2008 unter dem Übereinkommen über weiträumige grenzüberschreitende Luftverschmutzung (UN ECE-CLRTAP), NFR Inventartabellen. http://www.umweltbundesamt.de/emissionen/archiv/unece_clrtap_de_2008.zip 
UBA (2008c) Abfrage der UBA Grundwasserdatenbank, Persönliche Mitteilung des Umweltbundesamts via E-Mail

UMWELT (2006) Referat IG II 4 „Chemikalien, Risikobewertung und Risikomanagement", Magazin des Bundesministeriums für Umwelt, Naturschutz und Reaktorsicherheit (BMU). 4:245-246

Umweltbeobachtung (2007) UMEG, Zentrum für Umweltmessungen, Umwelterhebungen und Gerätesicherheit Baden-Württemberg, Atmosphärische Deposition der Jahre 2002 bis 2004 an den Intensiv-Messstellen in Baden-Württemberg. Europ. Env. eJournal http://www.umweltbeobachtung.de/journal//U811-MDBW11J02-de.pdf (abgerufen im November 2007)

Vink R, Behrendt H (2002) Emissions, immissions and retention processes of heavy metals in the Rhine and Elbe drainage areas and their tributaries. Hydrol process 16:3227-3244
Welker A (2004) Schadstoffströme im urbanen Wasserkreislauf Aufkommen und Verteilung, insbesondere in den Abwasserentsorgungssystemen, Habilitationsschrift. http://kluedo.ub.uni-kl.de/ volltexte/2005/1892/

Yang Y, Ligouis B, Pies C, Grathwohl P, Hofmann T (2008) Occurrence of coal and coal-derived particle-bound polycyclic aromatic hydrocarbons (PAHs) in a river floodplain soil. Environmental Pollution 151:121-129

Yunker MB, Macdonald RW, Vingarzanc R, Mitchell RH, Goyettee D, Sylvestrec S (2002) PAHs in the Fraser River basin: a critical appraisal of PAH ratios as indicators of PAH source and composition. Org Geochem 33:489-515

ZKR Zentralkommision für die Rheinschifffahrt (2008) Persönliche Mitteilung 